\title{
Doctor, How Much Weight Will I Lose?-a New Individualized Predictive Model for Weight Loss
}

\author{
André Goulart $^{1,2}$ • Pedro Leão $0^{1,2}$ • Patrício Costa ${ }^{2}$ - Maria Pereira ${ }^{3}$ - Aline Fernandes ${ }^{3}$. \\ Fernando Manso ${ }^{1}$. José Maia-da-Costa ${ }^{1}$
}

(C) Springer Science+Business Media New York 2016

\begin{abstract}
Bariatric surgery is an effective treatment for weight loss, but the patient's ability to reach a sustained weight loss depends upon several technical and individual factors. Creating an easy model that adapts bariatric surgery's weight loss goals for each patient is very important for presurgery and follow-up evaluations.
\end{abstract}

Keywords Bariatric surgery $\cdot$ Sleeve gastrectomy $\cdot$ Weight loss

\author{
José Maia-da-Costa \\ jmmaiadacosta@gmail.com \\ André Goulart \\ andre.b.goulart@gmail.com \\ Pedro Leão \\ pedroleao@ecsaude.uminho.pt \\ Patrício Costa \\ pcosta@ecsaude.uminho.pt \\ Maria Pereira \\ maria_lpereira@hotmail.com \\ Aline Fernandes \\ aline_fernandes_nut@hotmail.com \\ Fernando Manso \\ fjcmanso@hotmail.com
}

1 Department of General Surgery, Hospital de Braga, Sete Fontes, 4710-243 São Victor, Braga, Portugal

2 Life and Health Sciences Research Institute (ICVS), University of Minho, Braga, Portugal

3 Department of Endocrinology, Hospital de Braga, Braga, Portugal

\section{Introduction}

Bariatric surgery was developed with the purpose of being an effective treatment for sustained weight loss, improvement of obesity-related comorbidities such as hypertension, diabetes, and sleep apnea, and better quality of life for morbidly obese patients [1]. In spite of these potential benefits, bariatric surgery presents up to $20 \%$ of morbidity and less than $1 \%$ of mortality [2].

The measure of bariatric surgery success is controversial. The most widely used definition of success is $50 \%$ excess weight loss (\%EWL) [3]. However, 15 to $20 \%$ of the patients fail to accomplish this mark [4]. This static definition, established by consensus, is being called into question as it does not take into account individual patient factors. Recently, new definitions such as dynamic percentage total weight loss (\%TWL) charts for laparoscopic Roux-en-Y gastric bypass have been suggested [5].

Each patient's ability to reach a sustained weight loss depends upon several technical and individual factors [2]. Numerous studies have been conducted with the purpose of identifying predictive factors for weight loss in order to optimize the process of selection of patients to undergo bariatric procedures [2]. The main factors identified in these studies were body mass index (BMI) pre-surgery [6-9], BMI post-surgery [7], sex [8], age [8], diabetes [9], and psychiatric factors [10].

Despite these findings, no model to date can individually predict pre-operatively the expected weight loss after surgery. The creation of a model that adapts weight loss goals of bariatric surgery for each patient would undoubtedly be an important tool at pre-surgery and follow-up evaluations.

The aim of this work is to create an easy and personalized model that will be able to accurately predict 
the expected weight 1 year after laparoscopic sleeve gastrectomy ( $\mathrm{SG}$ ) and use it as a realistic patienttailored goal to achieve at follow-up consultations.

\section{Methods}

\section{Statistical Analysis and Ethical Approval}

A multiple linear regression model (MLRM) was developed using 1 year post-surgery BMI as the dependent variable and sex, age, pre-surgery BMI, hypertension, diabetes, dyslipidemia, and sleep apnea as independent variables/predictors.

To test the accuracy of the predictive model, a paired sample $t$ test was performed between the 1 year after surgery BMI estimates and the observed 1 year after surgery BMI.

All statistical analysis was performed using SPSS (IBM SPSS Statistics v. 20) and the results were considered to be significant for $p$ values $<0.05$.

The present study was submitted to the consideration by the Ethics Committee of Hospital the Braga and received approval.

\section{Model Development}

A retrospective analysis of the data from 152 patients submitted to SG at our institution between August 2008 and
September 2012 was performed in order to identify predictive factors of weight loss. All patients were submitted to primary SG (no previous bariatric surgery) and had a minimum of 1 year of follow-up.

The MLRM was significant $(\mathrm{F}(2152)=135.0 ; p<0.001)$ and the set of predictors explains $64 \%$ of the weight loss ( $R^{2}=0.640$; adjusted $\left.R^{2}=0.635\right)$. The significant predictors were pre-surgery BMI $(\beta=0.742 ; p<0.001)$ and age $(\beta=0.249 ; p<0.001)$ and, based on these, the equation for the model was determined with a standard error of estimate of 3.38. When controlling for pre-surgery BMI, age and gender, comorbidities did not show a significant effect on 1 year after surgery BMI.

The regression equation found is 1 year after surgery $\mathrm{BMI}=-3.597+0.621 \times$ pre-surgery $\mathrm{BMI}+0.135 \times$ age .

\section{Model Validation}

In order to validate this model, a prospective study was developed and all patients submitted to SG between June 2013 and May 2014 were included. Patients who were previously submitted to any bariatric procedure were excluded. Minimum duration of follow-up after surgery was 1 year. Data collection ended on May 2015.
Fig. 1 Correlation between BMI pre-surgery and BMI 1 year after surgery in respect to age categories $(\leq 40$ years and $>40$ years old)

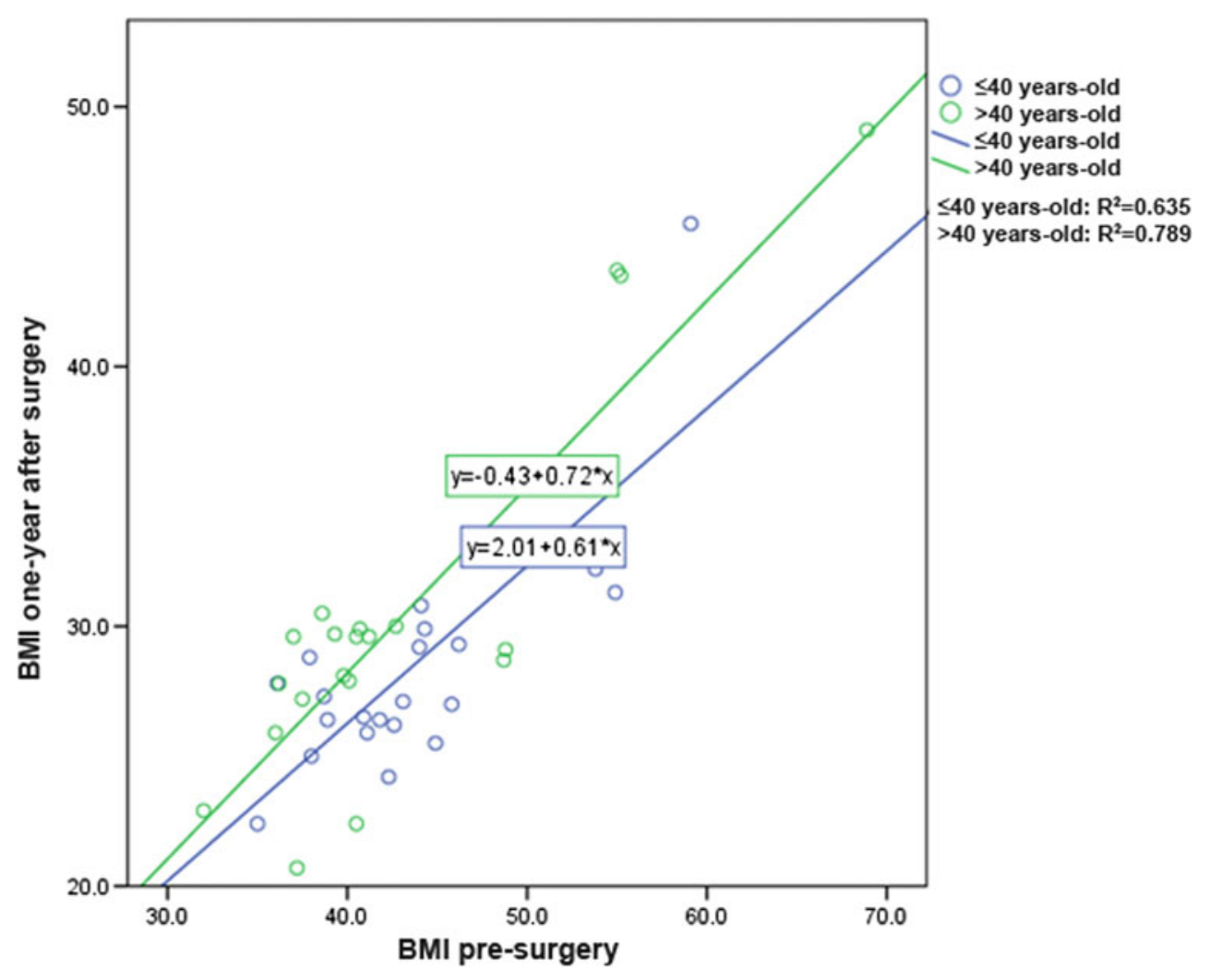




\section{Results}

The validation sample included 45 patients, primarily female $(89 \%)$ and all participants were Caucasian. Mean age was 41.9 years $( \pm 9.5$; range between 22 and 67 years) with 22 patients (49\%) 40 years or less and 23 patients $(51 \%)$ over 40 years old. Mean pre-surgery BMI measured by bioimpedance was $43.6 \mathrm{~kg} / \mathrm{m}^{2}$ ( \pm 7.4 ; range between 32 and $68.9 \mathrm{~kg} / \mathrm{m}^{2}$ ). All patients were submitted to laparoscopic SG.

The observed percentage of excess BMI lost (\%EBMIL) 1 year after surgery was $80.2 \%$ (standard deviation $21.8 \%$, minimum $38 \%$, maximum $135 \%$ ). The expected \%EBMIL evaluated by the model was $82.9 \%$ (standard deviation $16.7 \%$, minimum $53 \%$, maximum $126 \%$ ).

The estimates are normally distributed except for one patient that presented a z-score 3.2. Considering the normal curve $95 \%$ confidence interval boundaries (z-score \pm 1.96 ), we decided to exclude this patient from the validation sample.

The final model accurately predicted the 1 year postsurgery BMI $\left(R^{2}=0.756\right.$ : adjusted $R^{2}=0.745$; standard error $3.01)$. The predictive power of the model was also observed when two age groups were considered: subgroup $\leq 40$ years old $\left(R^{2}=0.635\right)$ and $>40$ years old $\left(R^{2}=0.789\right)$ (Fig. 1$)$.

The difference between the 1 year after surgery BMI estimates and the observed 1 year after surgery BMI was not statistically significant $(\mathrm{t}(43)=-1.054 ; p=0.298)$.

\section{Conclusion}

Most patients' desire is to know before-hand how much height they will lose after being submitted to a bariatric procedure (SG). A BMI of $25 \mathrm{~kg} / \mathrm{m}^{2}$ may not be an appropriate weight loss goal for most patients and setting this demanding targetweight can be very demotivating both for the patient (leading to drop-out from consultations and nutritional counseling as they feel it will never be accomplished) and the healthcare professional.

The present study validated a post-bariatric surgery BMI prediction model, confirming the results from our previous study that found that BMI pre-surgery and age were very good predictors of BMI 365 days post-surgery. The presented model was able to accurately predict BMI 1 year after surgery and no statistically significant difference was found between the BMI 1 year after surgery predicted by the model and that observed by the follow-up.

This simple model can be a very useful tool for the presurgery and follow-up consultations because it offers a realistic and personalized goal that can be used to motivate patients.

The present validation was set in a small sample of patients submitted to laparoscopic SG and despite the consistent results, this weight loss prediction formula needs replication in studies with larger sample and with different bariatric procedures to take its place as a powerful tool for bariatric surgery counseling.

Acknowledgments Joana Silva Monteiro, MD MSc.

Compliance with Ethical Standards The present study was submitted to the consideration by the Ethics Committee of Hospital the Braga and received approval.

Funding This study was not funded.

Conflict of Interest The authors declare that they have no conflict of interest.

Ethical Approval All procedures performed in studies involving human participants were in accordance with the ethical standards of the institutional and/or national research committee and with the 1964 Helsinki declaration and its later amendments or comparable ethical standards.

Informed Consent Informed consent was obtained from all individual participants included in the study.

\section{References}

1. Buchwald H, Avidor Y, Braunwald E, et al. Bariatric surgery: a systematic review and meta-analysis. JAMA. 2004;292(14):172437. doi:10.1001/jama.292.14.1724.

2. Livhits M, Mercado C, Yermilov I, et al. Preoperative predictors of weight loss following bariatric surgery: systematic review. Obes Surg. 2012;22(1):70-89. doi:10.1007/s11695-011-0472-4.

3. Oria HE, Moorehead MK. Bariatric analysis and reporting outcome system (BAROS). Obes Surg. 1998;8(5):487-99. doi:10.1381/ 096089298765554043.

4. Maggard MA, Shugarman LR, Suttorp M, et al. Meta-analysis: surgical treatment of obesity. Ann Intern Med. 2005;142(7):54759.

5. van de Laar AW, Acherman YI. Weight loss percentile charts of large representative series: a benchmark defining sufficient weight loss challenging current criteria for success of bariatric surgery. Obes Surg. 2014;24(5):727-34. doi:10.1007/s11695-013-1130-9.

6. Coupaye M, Sabate JM, Castel B, et al. Predictive factors of weight loss 1 year after laparoscopic gastric bypass in obese patients. Obes Surg. 2010;20(12):1671-7. doi:10.1007/s11695-010-0159-2.

7. Chen EY, McCloskey MS, Doyle P, et al. Body mass index as a predictor of 1-year outcome in gastric bypass surgery. Obes Surg. 2009;19(9):1240-2. doi:10.1007/s11695-008-9724-3.

8. Ma Y, Pagoto SL, Olendzki BC, et al. Predictors of weight status following laparoscopic gastric bypass. Obes Surg. 2006;16(9): 1227-31. doi:10.1381/096089206778392284.

9. Adams ST, Salhab M, Hussain ZI, et al. Roux-en-Y gastric bypass for morbid obesity: what are the preoperative predictors of weight loss? Postgrad Med J. 2013;89(1053):411-6. doi:10.1136/ postgradmedj-2012-131310. quiz 5, 6 .

10. Sockalingam $\mathrm{S}$, Cassin $\mathrm{S}$, Crawford SA, et al. Psychiatric predictors of surgery non-completion following suitability assessment for bariatric surgery. Obes Surg. 2013;23(2):205-11. doi:10.1007/s11695012-0762-5. 